\title{
Dependência química induzida pelo uso de benzodiazepínicos na senescencia
}

\author{
Chemical dependency induced by the use of benzodiazepines in senescence \\ Dependencia química inducida por el uso de benzodiazepinas en la senescência
}

Recebido: 26/10/2021 | Revisado: 05/11/2021 | Aceito: 06/11/2021 | Publicado: 10/11/2021

\author{
Adrielle Aquino da Silva \\ ORCID: https://orcid.org/0000-0002-9572-4946 \\ Universidade Nilton Lins, Brasil \\ E-mail: aquinoadrielle@gmail.com \\ Gabriel Oliveira de Souza \\ ORCID: https://orcid.org/0000-0001-9168-074X \\ Universidade Nilton Lins, Brasil \\ E-mail: gabriel.souza@uniniltonlins.edu.br
}

\begin{abstract}
Resumo
Devido aos efeitos sedativos e tranquilizante os idosos utilizam por tempo além do recomendado, ocasionando muita das vezes efeitos indesejados, como é o caso da dependência. Em torno disso, o objetivo deste trabalho por meio de revisão, foi levantar as causas da toxicologia e dependência, além do entendimento sobre o manejo da retirada do fármaco. Metodologia: Foram utilizadas as plataformas SCIELO, LILICS, MEDLINE/PUBMED e livro, onde passaram por critérios de exclusão e inclusão, resultando em 35 estudos incluídos. Resultado e Discussão: Os BZDs são medicamentos no geral seguros, porém se utilizados por curtos prazos, sendo os mais recomendados para idosos a utilização de BZDs com meia vida curta. A grande parte da toxicologia são cognitivos e psicomotores, além da dependência. Para o manejo da dependência são utilizados a redução gradual ou auxilio com outras classes medicamentosas. Considerações finais: O presente estudo mostra de forma objetiva e clara a toxicologia nos idosos, dependência e manejo da dependência, sendo umas das dificuldades para o manejo o aparecimento de sintomas que se parecem com o retorno das causas para o início da terapia medicamentosa.
\end{abstract}

Palavras-chave: Benzodiazepínicos em idosos; Toxicologia dos benzodiazepínicos; Dependência na senescência.

\begin{abstract}
Due to the sedative and tranquilizer effects, the elderly use for longer than recommended, causing many unwanted effects, such as dependence. Around this, the objective of this work, by means of review, was to raise the causes of toxicology and dependence, in addition to understanding the management of drug withdrawal. Methodology: The SCIELO, LILICS, MEDLINE/PUBMED platforms and book were used, where they went through exclusion and inclusion criteria, resulting in 35 included studies. Results and Discussion: BZDs are generally safe drugs, but if used for short periods, being the most recommended for the elderly the use of BZDs with a short half-life. The great part of toxicology is cognitive and psychomotor, besides dependence. For the management of dependence, gradual reduction or assistance with other drug classes are used. Final considerations: The present study shows objectively and clearly the toxicology in the elderly, dependence and management of dependence, is one of the difficulties for the management the appearance of symptoms that resemble the return of the causes for the beginning of drug therapy.
\end{abstract}

Keywords: Benzodiazepines in the elderly; Benzodiazepines toxicology; Dependence on senescence.

\section{Resumen}

Debido a los efectos sedantes y tranquilizantes, los ancionos lo usan durante más tiempo de lo recomendado, lo que a menudo provoca efectos no deseados, como la adcción. En torno a esto, el objetivo de este trabajo, a través de una revisión, fue plantear las causas de la toxicología y la adicción, además de compreender el manejo de la abstinencia de drogas. Metodología: se utilizaron SCIELO, LILICS, MEDLINE/PUBMED y plataformas de libros, donde pasaron po criterios de exclusión e inclusión, resultando en 35 estudios incluidos. Resultados y discusión: Las BZD son generalmente medicamentos seguros, pero si se usan por períodos cortos, lo recomendado para los ancianos es el uso de BZD con una vida media corta. Gran parte de la toxicología es cognitiva y psicomotora, además de la adicción. Para manejar la dependencia, se utiliza la reducción gradual o la asistencia con otras clases de drogas. Consideraciones finales: El presente estudio muestra de manera objetiva y clara la toxicología en el anciano, la dependencia y el manejo de la dependencia, siendo una de las dificultades para el manejo la aparición de sintomas que asemejan el retorno de causas para el inicio de la farmacoterapia.

Palabras clave: Benzodiazepinas en los ancianos; Toxicología de las benzodiazepinas; Dependencia de la senescencia. 


\section{Introdução}

Desde a década de 1960 os BZDs são utilizados na prática clínica, como substituto dos Barbitúricos, e acabaram se tornaram bastante populares, pois sua ação terapêutica reduz os níveis de ansiedade, agem como anticonvulsivantes e miorrelaxantes, são utilizadas como indução de anestésicos e para tratamento de síndromes de abstinência por álcool. (Vinkers \& Olivier, 2012; Danza et al., 2015). Essa classe de medicação atua no receptor ácido gama aminobutírico (GABA) inibindo a excitabilidade necessária para a sinapse entre neurônios, essa inibição ocorre quando os BZDs se ligam a subunidades $\alpha$ e y do GABA e induzem a uma mudança na conformação do canal de cloreto, ocasionando a hiperpolarização do receptor, levando assim a inibição e consequentemente a redução da excitabilidade neural, ocasionando os efeitos desejados (Griffin et al., 2013).

Devido as propriedades sedativas/tranquilizantes, os idosos utilizam como estratégia para lhe dar com o nervosismo, como alivio dos distúrbios de sono, ansiedade e a solidão. Diante dessa sensação de alivio, os BZDs acabam se tornando “essenciais” para encarar o cotidiano (Alvarenga et al, 2014). Apesar das diretrizes de boas práticas sugerirem que a duração de uso deve ser de algumas semanas, muitas pessoas utilizam por anos, tornando o uso crônico (Gage et al., 2012).

Apesar dos BZDs serem úteis para o tratamento de insônia e ansiedade, os números de resultados adversos têm sido crescentes, principalmente em idosos, com risco de até 50\% de demência (Gage et al., 2012). Além de causarem desorientação, comprometimento cognitivo e psicomotor (Lader, 2014) e quando utilizadas coadministradas com outros agentes, como medicamentos ou etanol, podem levar a uma depressão respiratória (Kang, Galuska \& Ghassemzadeh, 2021).

A duração para tratamento com BZDs é aconselhado que seja limitado de 2 a 4 semana, reduzindo de forma gradual a dosagem, porém na prática o uso prolongado é observado frequentemente, o que pode acarreta dependência, que resulta em tolerância e síndrome de abstinência, se retirado o uso de forma abrupta (Canham, Gallo \& Simoni, 2014; Vinckenbosch et al., 2021). A tolerância está relacionada com alterações nos receptores alvos dessa substância, levando a queda dos efeitos farmacológicos, e podem causar a abstinência que possui sintomas que podem ser confundidos com o retorno da ansiedade (Schallember \& Colet, 2016).

Vale ressaltar que as crises de abstinência podem variar de acordo com o processo farmacocinético da medicação, os fármacos com meia vida longa são eliminados de forma mais lenta, ficando disponível no organismo por mais tempo, ocasionando uma crise de abstinência com poucos sintomas físicos, já os fármacos com meia vida curta, ficam disponíveis em uma pequena quantidade de tempo e causam crises com sintomas mais intensos (Katzung, Masters \& Trevor, 2014).

Jacob, Raapp e Kostev (2017), relataram em seus estudos que o uso dos BZDs a longo prazo em idosos tem um aumento conforme a idade, levantando a estatística de cerca de 12,3\% de idosos entre 65 - 70 anos; 15,5\% para idosos de 71 - 80; $23,7 \%$ com idade de 81 - 90 e 31, 6\% para idosos com mais de 90 anos. Já Torres-Bondia et al. (2020), demonstrou em sua pesquisa que $36,1 \%$ de pessoas com mais de 65 anos utilizam os BZDs a longo prazo.

Os padrões de uso prologando dos BZDs em idosos tem sido crescente, apesar da indicação do uso serem para tratamentos a curto prazo. Com a alta prevalência de uso a longo prazo dos BZDs, tende a ter um aumento significativo de dependência entre idosos e efeitos toxicológicos não desejáveis. Levando em consideração todo o contexto já exposto, tornouse necessário o levantamento das causas da dependência, da toxicologia causadora de vários comprometimentos em idosos e acerca do manejo da dependência.

\section{Metodologia}

Trata-se de uma revisão integrativa, que segundo Botelho, Cunha \& Macedo (2011), é um método especifico que resume experiências anteriores ou literatura teórica, fornecendo uma compreensão mais abrangente de um assunto específico, visando analisar e sintetizar vários estudos publicados para geração de novos conhecimentos com base nos resultados dos estudos 
anteriores, para preparar uma revisão integrativa é necessário que as etapas sejam descritas de forma clara, etapas como a identificação do tema, estabelecimento dos critérios de exclusão e inclusão, identificação de estudos pré-selecionados e selecionados, categorização dos estudos, analise e interpretação dos resultados e apresentação da revisão/síntese do conhecimento.

Seguindo o método de revisão relado, foi desenvolvida através de uma abordagem qualitativa com o tipo de pesquisa descritiva, utilizando-se de fontes secundarias para contextualizar a toxicologia dos benzodiazepínicos, a dependência do uso a longo prazo em idosos e o manejo da dependência. Para tanto, usou-se de pesquisas em livros e nas plataformas Scientific Electronicc Library Online - SCIELO, Medical Literature Analysis and Retrievel System Online - MEDLINE/PUBMED e Literatura Latino-Americana e do Caribe em Ciências da Saúde - LILICS.

Com relação ao procedimento, foram utilizadas de forma associada os descritores: "Benzodiazepínicos", "Mecanismo de ação" "Dependência", “Toxicologia" e "Idosos" "Manejo Medicamentoso", nos idiomas: português, inglês e espanhol. Os estudos obtidos na busca nos meses de agosto a outubro de 2021, passaram pelos critérios de exclusão, onde os que não preenchiam os critérios de elegibilidade foram excluídos tais como, trabalhos fora do tempo proposto (2011 a 2021), sem a temática abordada e repetidos nas bases de dados, como mostra a Figura 1.

Os critérios de inclusão foram: Artigos coerentes ao tema disposto, publicados dentro do tempo estabelecido, estudos completos e de acesso livre. Desda forma deu-se seguimento da revisão com base na elaboração de quadro sinóptico contendo: Ano, autor, título, objetivos e principais resultados.

Figura 1. Fluxograma de critérios de exclusão e inclusão.

\section{Estudos identificados (240)}
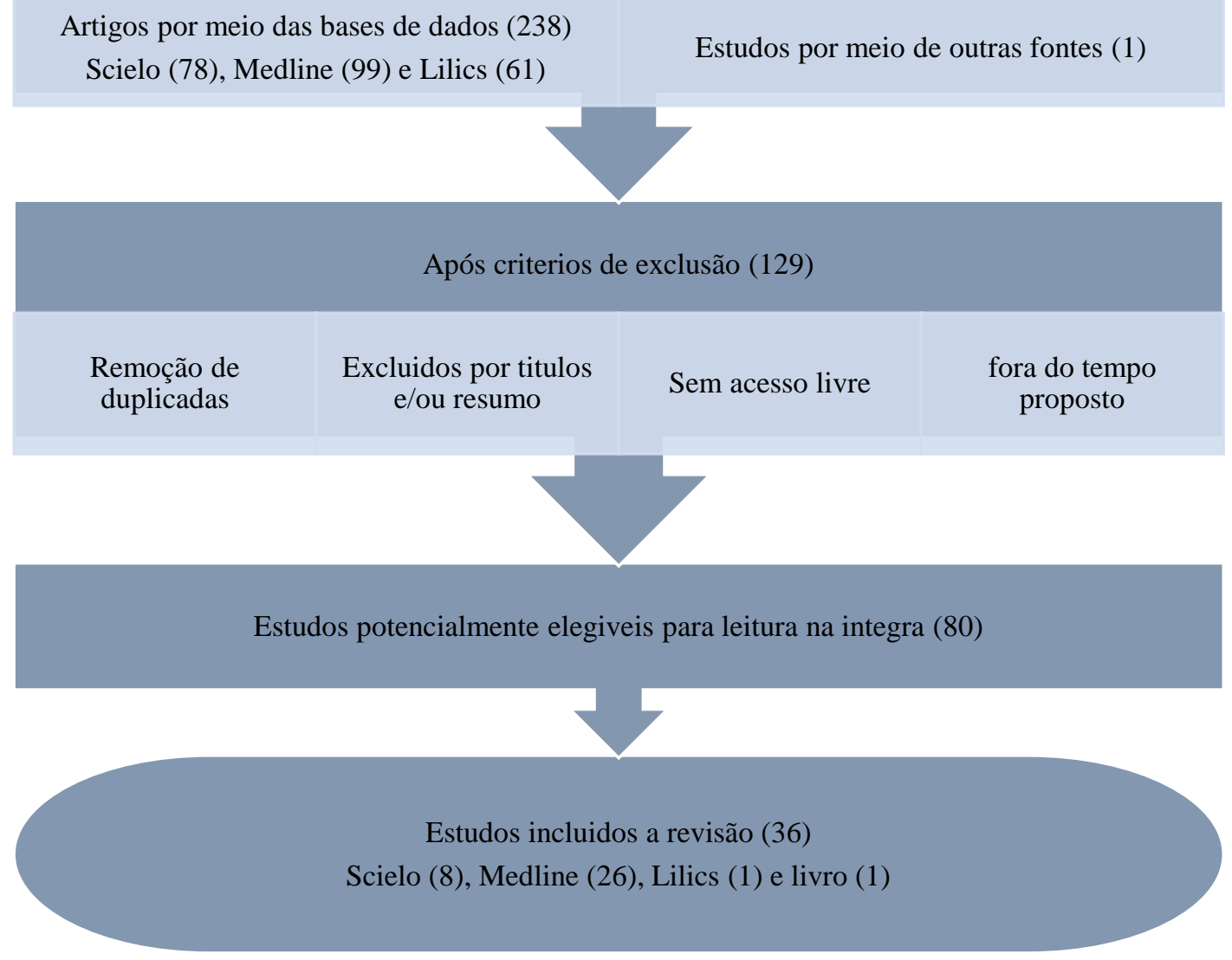

Fonte: Autores (2021). 


\section{Resultados e Discussão}

Diante dos resultados encontrados após os critérios de exclusão e inclusão, desenvolveu-se um quadro com as características dos principais artigos selecionados, como descrito a seguir no Quadro 1:

Quadro 1. Características dos artigos analisados.

\begin{tabular}{|c|c|c|c|}
\hline $\begin{array}{l}\text { AUTOR, } \\
\text { ANO }\end{array}$ & TíTULO & OBJETIVOS & PRINCIPAIS RESULTADOS \\
\hline $\begin{array}{l}\text { Vinckencos } \\
\text { ch } \text { et al., } \\
2021\end{array}$ & $\begin{array}{l}\text { Na explorative approch to understanding } \\
\text { indicidual diferences in driving } \\
\text { performance and neurocognition on } \\
\text { long-term benzodiazepine users }\end{array}$ & $\begin{array}{l}\text { Explorar o papel da } \\
\text { intoxicação aguda e das } \\
\text { queixas clinicas. }\end{array}$ & $\begin{array}{l}\text { Os usuários de BZDstiveram desempenho pior em tarefas de } \\
\text { velocidade de resposta, velocidade de processamento e } \\
\text { atenção sustentada. }\end{array}$ \\
\hline $\begin{array}{l}\text { Lukacisino } \\
\text { vá et al., } \\
\quad 2021\end{array}$ & $\begin{array}{l}\text { The prevalence and prescribing patterns } \\
\text { of benzodiazepines and Z-drugs in older } \\
\text { nursing home residents in diferente } \\
\text { European contries and Israel: } \\
\text { retrospective results from the eu shelter } \\
\text { study }\end{array}$ & $\begin{array}{l}\text { Determinar e comparar a } \\
\text { prevalência e os padrões de } \\
\text { prescrição de BZDs/Z-drogas } \\
\text { em residentes de lares de } \\
\text { idosos. }\end{array}$ & $\begin{array}{l}\text { A prevalência variou de } 44,1 \% \text { em Israel a } 14,5 \% \text { na } \\
\text { Alemanha. Os BZDs frequentemente prescritos foram } \\
\text { Lorazepam, Oxazepam e Brotizolam, enquanto que os Z- } \\
\text { drogas foram representados principalmente por Zopiclona e } \\
\text { Zolpidem. }\end{array}$ \\
\hline $\begin{array}{l}\text { Benini } e t \\
\text { al., } 2021\end{array}$ & $\begin{array}{l}\text { Continuous infusion of flumazenil in the } \\
\text { management of benzodiazepines } \\
\text { detoxification. }\end{array}$ & $\begin{array}{l}\text { Confirmar a eficácia do } \\
\text { Flumazenil na redução da } \\
\text { síndrome de abstinência dos } \\
\text { BZDs. }\end{array}$ & $\begin{array}{l}\text { Identificaram que as concentrações séricas de Flumazenil } \\
\text { diminuíram após } 4 \text { dias de tratamento, concluindo que a } \\
\text { influsão elastomerica de Flumazenil para desisntoxicação de } \\
\text { BZDs é um dispositivo viável para manter as concentrações } \\
\text { adequadas, constantes e toleradas para a redução. }\end{array}$ \\
\hline $\begin{array}{l}\text { Tseng et } \\
\text { al., } 2019\end{array}$ & $\begin{array}{l}\text { Benzodiazepines, Z-hypnotics, and risk } \\
\text { of dementia: special consoderations of } \\
\text { half-lives and concomitante Use }\end{array}$ & $\begin{array}{l}\text { Investigar a associação entre } \\
\text { BZDs ou z-hipnóticos e o } \\
\text { risco de demência, além de } \\
\text { testar a hipótese de que as } \\
\text { diferentes meia-vidas podem } \\
\text { impactar no risco de } \\
\text { demência. } \\
\end{array}$ & $\begin{array}{l}\text { Identificaram que os BZDs de curta ação e Z-hipnóticos } \\
\text { estavam associados a um maior risco de demência do que os } \\
\text { usuários de longa ação, com risco ainda mais intensificado } \\
\text { para usuários de } 2 \text { ou mais BZDs ou Z-hipnóticos. }\end{array}$ \\
\hline $\begin{array}{l}\text { Baandrup } \\
\text { et al., } 2018\end{array}$ & Pharmacological interventions for benz & $\begin{array}{l}\text { Avaliar os benefícios e } \\
\text { malefícios das intervenções } \\
\text { farmacológicas para facilitar } \\
\text { a descontinuação do uso }\end{array}$ & $\begin{array}{c}\text { As seguintes intervenções com Carbamazepinna, Pregabalina, } \\
\text { Captodiame, Paroxetina e Flumazenil, reduzem os sintomas de } \\
\text { ansiedade e abstinências tem evidências de qualidades baixas, } \\
\text { não sendo possível uma conclusão firme sobre as intervenções } \\
\text { farmacológicas para a facilitação da descontinuidade. }\end{array}$ \\
\hline $\begin{array}{l}\text { Fluyau; } \\
\text { Revadigar; } \\
\text { MAnobianc } \\
\text { o, } 2018\end{array}$ & $\begin{array}{l}\text { Challenges of the pharmacological } \\
\text { management of benzodiazepine } \\
\text { withdrawal dependence and } \\
\text { discontinuantion }\end{array}$ & $\begin{array}{l}\text { Revisão acerca do manejo } \\
\text { farmacológico da } \\
\text { descontinuação dos BZDs, } \\
\text { ampliando a eficácia e os } \\
\text { riscos de dependência, } \\
\end{array}$ & $\begin{array}{l}\text { A eficácia desses medicamentos não é robusta, apesar de } \\
\text { serem relativamente seguros, alguns deles tem um índice } \\
\text { terapêutico estreito, efeitos colaterais graves e potencialmente } \\
\text { fatais. }\end{array}$ \\
\hline $\begin{array}{l}\text { Schallembe } \\
\text { rger; Colet, } \\
2016\end{array}$ & $\begin{array}{l}\text { Assessnebt of dependence and anxiety } \\
\text { among benzodiazepine users in a } \\
\text { provincial municipality in Rio Grande } \\
\text { do Sul, Brazil }\end{array}$ & $\begin{array}{l}\text { Avaliação dos níveis de } \\
\text { dependência e ansiedade em } \\
\text { usuários dessas drogas no } \\
\text { sistema público de saúde. }\end{array}$ & $\begin{array}{c}\text { A avaliação da dependência indicou que mais da metade dos } \\
\text { usuários eram dependentes de BZDs e a maioria apresentava } \\
\text { um grau severo de ansiedade. }\end{array}$ \\
\hline $\begin{array}{l}\text { Janhsen; } \\
\text { Roser; } \\
\text { Hoffmann, } \\
\quad 2015\end{array}$ & $\begin{array}{c}\text { The problems of long-term treatment of } \\
\text { Withdrawal }\end{array}$ & $\begin{array}{l}\text { Fornecer uma visão geral das } \\
\text { taxas de abuso e dependência } \\
\text { de BZDs e Z-hipnóticos, } \\
\text { além de explicar como } \\
\text { realizar o tratamento de } \\
\text { abstinência e discutir a } \\
\text { pratica de prescrição. } \\
\end{array}$ & $\begin{array}{l}\text { As estimativas do número de usuários de BZDs variam de } \\
\text { 128mil a 1,6 milhões na Alemanha. Para o tratamento } \\
\text { ambulatorial da abstinência a recomendação é que haja uma } \\
\text { troca por uma dose equivalente de outro BZD com efeito } \\
\text { prolongado, sendo reduzido semanalmente. }\end{array}$ \\
\hline $\begin{array}{l}\text { Gage et al., } \\
\quad 2014\end{array}$ & $\begin{array}{l}\text { Benzodiazepine use and risk of } \\
\text { Alzheimer's disease: case-control study }\end{array}$ & $\begin{array}{l}\text { Investigar a relação entre o } \\
\text { risco da doença de Alzheimer } \\
\text { e a exposição aos BZDs } \\
\end{array}$ & $\begin{array}{c}\text { Houve uma associação aumentada com a densidade de } \\
\text { exposição, e com a meia-vida do medicamento, } \\
\text { principalmente com os BZDs de longa ação. }\end{array}$ \\
\hline $\begin{array}{l}\text { Gage et al., } \\
\quad 2012\end{array}$ & $\begin{array}{l}\text { Benzodiazepine use and risk of } \\
\text { dementia: prospective population based } \\
\text { study }\end{array}$ & $\begin{array}{l}\text { Avaliar a associação entre o } \\
\text { uso de BZDs e o incidente de } \\
\text { demência }\end{array}$ & $\begin{array}{l}\text { Durante o acompanhamento de } 15 \text { anos em pacientes com } \\
\text { idade média de } 78,2 \text { anos, o uso de BZDS foram associados a } \\
\text { riscos aumentados de demência. }\end{array}$ \\
\hline $\begin{array}{l}\text { Filho et al., } \\
\quad 2011\end{array}$ & $\begin{array}{l}\text { Utilização de BZDs por idosos de uma } \\
\text { estratégia de saúde da família: } \\
\text { implicações para enfermagem }\end{array}$ & $\begin{array}{c}\text { Verificar e analisar a } \\
\text { utilização dessa classe } \\
\text { medicamentosa por idosos } \\
\text { cadastrados em uma unidade } \\
\text { de estratégia da família do } \\
\text { município de Diamantina - } \\
\text { MG. }\end{array}$ & $\begin{array}{l}\text { Destacaram-se a faixa etária de } 71 \text { a } 75 \text { anos, do gênero } \\
\text { feminino e com escolaridade incompleta de primeiro grau. Os } \\
\text { medicamentos mais utilizados foram: Diazepam, Clonazepam, } \\
\text { Bromazepam e Alprazolam. }\end{array}$ \\
\hline
\end{tabular}

Fonte: Autores (2021). 


\subsection{Relação do receptor GABAA com os benzodiazepínicos}

O principal sistema neurotransmissor de inibição rápida no cérebro é os receptores $\mathrm{GABA}_{\mathrm{A}}$, que são formados por cinco subunidades transmembrana, juntos formam o canal de cloreto dependente de ligante. Esses receptores $\mathrm{GABA}_{\mathrm{A}}$ possuem subunidades típicas, sendo composta por duas subunidades $\alpha$, duas $\beta$ e uma subunidade y. Para que ocorra a abertura dos canais de cloreto com carga negativa $\left(\mathrm{Cl}^{-}\right)$é necessário que haja uma ligação de GABA ao receptor $\mathrm{GABA}_{\mathrm{A}}$ entre as subunidades $\alpha$ e $\beta$, iniciando assim um sinal inibitório (Vinkers \& Olivier, 2012).

Os benzodiazepínicos atuam entre as subunidades $\alpha$ e y, como moduladores alostéricos positivos (PAM) no domínio extracelular, agindo como co-agonista do GABA (Olsen, 2018), portando os BZDs ao se ligarem no seu local de ligação induzem a uma mudança na conformação do canal de cloreto, potencializando a ligação do GABA ao $\mathrm{GABA}_{\mathrm{A}}$, resultando em uma frequência maior dos canais, que leva uma maior entrada de íons de $\mathrm{Cl}^{-}$para o meio intracelular (IC), ocasionando a hiperpolarização do meio (Griffin et al., 2013).

É importante destacar que a subunidade $\alpha$ possuem seis subtipos, onde os BZDs se ligam a receptores $\mathrm{GABA}_{\mathrm{A}}$ que contenha os subtipos $\alpha_{1}, \alpha_{2}, \alpha_{3}$ ou $\alpha_{5}$ em combinação com a subunidades y, não interagindo aos que contenham os subtipos $\alpha_{4} \mathrm{e}$ $\alpha_{6}$, essas subunidades com subtipos diferentes contribuem com os diferentes efeitos dos BZDs (Vinkers \& Oliver, 2012). Sendo os subtipos $\alpha_{2}$ e $\alpha_{3}$ responsáveis pelo efeito ansiolítico, e os responsáveis pela sedação, amnesia e ataxia, são os $\alpha_{1}$ e $\alpha_{5}$ (Calcaterra $\&$ Barrow, 2014).

\subsection{A farmacocinética dos benzodiazepínicos em idosos}

Absorção, distribuição, metabolismo e excreção são etapas do processo de farmacocinética em que determina o início e duração do efeito dos medicamentos no organismo, essa ação pode variar de indivíduo a indivíduo devido a fatores como doenças preexistentes e alterações fisiológicas relacionadas à idade, principalmente no processo de meia-vida de eliminação do medicamento. Os BZDs são drogas medicamentosas bem absorvidas pelo trato gastrointestinal (TGI), possuem características de lipossolubilidade, além de serem altamente ligantes a proteínas plasmáticas, essas características influenciam no processo de distribuição. Por serem ligantes as proteínas, os BZDs após distribuídos no corpo, se acumulam em áreas ricas em tecidos adiposos e em lipídios, que é o caso do Sistema Nervoso Central. Além do mais, alguns BZDs desencadeiam uma ação adicional, devido aos metabolitos ativos gerados na fase I do ciclo de metabolismos hepático dos fármacos (Griffin et al., 2013).

Já no idoso ocorre modificações em alguns processos, ocasionados pela baixa na massa magra, albumina, água corporal e pelo aumento do tecido adiposo (Danza et al., 2015), com todas essas mudanças a um aumento no volume de distribuição, diminuição do metabolismo e excreção dos BZDs (Neft et al., 2019; Dominguez, 2016).

A redução do metabolismo pode ser contribuída pela redução do volume hepático, ocasionado pela redução do fluxo sanguíneo hepático, que respectivamente leva a diminuição das atividades da enzima citocromo P450 presente no fígado. Portando, o metabolismo hepático de fase I é realizada de forma reduzida (Tajiri \& Shimizu, 2013).

A depuração (excreção do fármaco) é realizada ainda no metabolismo hepático, na reação de fase II os metabolitos passam por um processo de conjugação para poderem ser eliminados através da urina, porém cada fármaco possui sua particularidade nesse processo, devido aos metabolitos ativos gerados por cada um (Katzung, Masters \& Trevor, 2014).

Portando, a forma em que o medicamento é manuseado pelo organismo sofre alterações fisiológicas resultadas do envelhecimento, sofrendo alterações no metabolismo, volume de distribuição e na diminuição da eliminação do fármaco, ocasionando um tempo de vida prolongado no organismo, com isso tem um aumento a toxicidade (Davies \& O`Mahony, 2015). 


\subsection{Toxicologia dos benzodiazepínicos em idosos}

Os BZDs estão associados a danos e efeitos adversos, porém a intensidade desses danos e efeitos podem variar de acordo com a concentração de pico e conforme a duração dos BZDs, além de que o envelhecimento torna os idosos mais sensíveis, podendo ter como causa uma redução de neurônios e respectivamente de receptores, tornando os receptores exposto por mais tempo a medicação (Lader, 2014), causando um nível de sedação maior do que o esperado, confusões mentais, fadiga e desempenho psicomotor com comprometimentos (Neft et al., 2019), como o aumento de riscos de quedas prejudiciais, com presença de lesões (Jiang et al., 2019)

Cunha et al. (2015), relataram que os BZDs mais utilizados foram Clonazepam, Diazepam, Alprazolam e Bromazepam. Já Filho et al. (2011), durante suas pesquisas identificou os BZDs mais utilizados, em primeiro lugar está o uso de Diazepam com 37,04\%, seguido de Clonazepam, Bromazepam, Alprazolam, Lorazepam e Midazolam. A prevalência do uso dos fármacos Diazepam e Clonazepam no Brasil, é derivada da distribuição gratuitamente do Programa Nacional de Assistência Farmacêutica.

Podemos citar o Diazepam e os seus efeitos adversos mais comuns em relação a esse fármaco, que são amnesia, tontura, ataxia e confusão, já em efeitos mais graves, como parada respiratória e convulsões prolongadas, são geralmente consequências de interações com opiáceos e álcool. Os efeitos adversos, principalmente em idosos tem relação com a meia-vida e com a fração livre do medicamento, que após a metabolização do diazepam, é gerado o metabolito ativo Desmetildiazepam, que se acumula a maior parte de doses circulantes no organismo e ficando disponíveis até 3 semanas (Calcaterra \& Barros, 2014). Para prevenir o acúmulo de substâncias em pessoas idosas e o aparecimento de reações adversas a medicamentos é recomendado a substituição por BZDs já hidroxilados e com meia vida de eliminação mais curta, que é o caso do Lorazepam, tendo um perfil de segurança maior em comparação os outros BZDs (Vila et al., 2012; Pomara et al., 2014).

Tseng et al. (2019), categorizou os benzodiazepínicos de acordo com a duração de ação para que houvesse uma relação de meia-vida com a demência, são eles de ação prolongada e de ação curta (Quadro 2), no entanto as duas categorias tiveram um risco aumentado de demência, 47\% maior para os de longa ação e 98\% para os de ação curta. Relataram também que os idosos que utilizavam mais de uma classe de sedativos tinha um risco ainda maior de desenvolvimento de demência do que os idosos que utilizava apenas uma única classe.

Quadro 2. Meia-vida dos Benzodiazepínicos.

\begin{tabular}{|c|c|c|}
\hline Meia-Vida & Medicamento & Meia-Vida em Horas \\
\hline Longa ação & Bromazepam & 20,6 \\
\hline Longa ação & Clonazepam & $30-40$ \\
\hline Longa ação & Diazepam & 48 \\
\hline Ação curta & Alprazolam & $10,7-15,8$ \\
\hline Ação curta & Lorazepam & $12-14$ \\
\hline Ação curta & Midazolam & $1,8-6,4$ \\
\hline
\end{tabular}

Fonte: Adaptado de TSENG et al. (2020)

Já estudos de Gage et al. (2014), demonstraram que o uso de BZDs foram significativamente relacionados a um risco aumentando de doença de Alzheimer, tendo associação maior com o uso de BZDs de ação mais longa do os que utilizaram os de curta ação. O risco de doença de Alzheimer teve aumento em 43 a 51\% entre pessoas que utilizaram benzodiazepínicos, esse aumento tem correlação com a intensidade de exposição e a utilizados BZDs de ação longa, porém não se pode descartar que o uso dos BZDs pode ser um marcador para o risco aumentando e não a causa em si. 


\subsection{A dependência dos benzodiazepínicos e o manejo da descontinuação em idosos}

A dependência dos BZDs é conhecida a pelo menos 40 anos, um fator que pode desencadeia essa dependência é uso terapêutico a longo prazo. Algumas diretrizes estabelecem o padrão de uso de 2 a 4 semanas (Marsden et al., 2019; Janhsen, Roser \& Hoffmann, 2015). Para Hood et al. (2014), o uso dessa substância mesmo usadas entre 3 a 6 semanas pode levar a dependência, sendo o desenvolvimento dessa condição bem semelhantes aos de outras classes medicamentosas.

Para compreender o mecanismo da dependência, é necessário saber que a área tegmental ventral (ATV), presente no mesencéfalo, mas também que inerva uma grande parte de regiões no cérebro, é composta de 70\% de neurônios dopaminérgicos, $15 \%$ de interneuronios GABAérgicos e 15\% de neurônios gluatamatérgicos. Sendo a subunidade $\alpha_{3}$ prevalente nos receptores $\mathrm{GABA}_{\mathrm{A}}$ dos neurônios dopaminérgicos AVT, enquanto que $\alpha_{1}$ é prevalente nos interneurônios. Com base nisso, os efeitos dos BZDs no circuito AVT e núcleo accumbens (NAC), que é uma via de recompensa, é determinada pelo seu efeito sobre a subunidade $\alpha_{1}$, prevalente no interneuronios GABAérgicos, a partir disso os interneuronios deixam de liberar GABA aos neurônios dopaminérgicos, causando uma perda de inibição, o que resulta a liberação descontrolada de dopamina no NAC (Rosas-Gutiérrez, Simón-Arcei \& Mercado, 2013).

A dependência pode causar tolerância e crises de abstinência, uma possível causa da tolerância pode estar relacionada com uma alteração nos receptores dessa substância, porém o mecanismo de ação da tolerância pode ser um processo mediado por vários mecanismos, sendo as teorias sobre esse mecanismo ainda conflitantes e sem possiblidades de validar um real mecanismo para essa condição (Canham, Gallo \& Simoni, 2014; Schallember \& Colet, 2016; Hood et al., 2014). Já no quadro de abstinência podem aparecer sintomas físicos e psicológicos, apresentando sintomas semelhantes ao que levou a iniciação terapêutica dos BZDs, por exemplo: câimbras musculares, irritabilidade, insônia, ansiedade e despersonalização. Portando a grande dificuldade atualmente na descontinuação desse fármaco, está na dependência e nos sintomas de abstinência, que 3apresenta uma mistura de sintomas originais que podem acontecer de forma mais intensa (Baandrup et al., 2018).

Para a descontinuação dos BZDs é recomendado que ocorra esse processo a longo tempo, para prevenção de complicações, como convulsões, confusão e delírio. Pode se utilizar de interversões de prescrição, como a substituição do fármaco ou a redução do benzodiazepínico para um oitavo da dose em um tempo de 2 semanas, sendo apropriado a redução da quantidade gradualmente no intervalo de 4 a 6 semanas. Uma alternativa para atuar como terapia adjuvante da redução gradual é a Carbamezepina, que apresentou benefícios em ensaios controlados, no entanto apresenta efeitos colaterais altos. Por outro lado, estudos concluíram que a transferência gradual para o uso de Diazepam é eficaz para a descontinuação, compensando precocemente a abstinência, já que seu tempo no organismo é prolongado, porém deve-se ter atenção na prescrição do mesmo, já que nos idosos o metabólito ativo Desmetildiazepam se acumula significativamente (Fluyau, Revadigar \& Manobianco, 2018).

Não existem comprovações suficientes para sustentar o uso de um BZD para redução gradual de doses em adultos mais velhos, porém é recomendado a troca por um BZD de longa ação, como é o caso de Diazepam ou Clonazepam, seguindo semanalmente com a diminuição gradual da dose, sendo reduzido de 10 a $25 \%$ da dose ao decorrer de 4 a 10 semanas, fazendose necessário uma abordagem de retirada bem mais lenta para pacientes que receberam terapia a longo prazo com BZDs, pois existe uma resistência à redução de dose (Reeves \& Kamal, 2019; Janhsen, Roser \& Hoffmann, 2015).

Benini et al. (2021), cita que existe um tratamento comum para síndrome de abstinência dos BZDs, que inclui de forma individual ou combinada: a redução gradual da droga; uma mudança para uma dose equivalente, porém de um benzodiazepínico de meia vida longa, antes da retirada; e a utilização de medicamentos antes da desintoxicação, dando continuidade após a descontinuação do BZDs. O Flumazenil é considerado um antagonista dos BZDs, sendo indicado para a desintoxicação rápida, além da redução dos sintomas de abstinência quando administradas em várias infusões lentas em bolus (1mg em 5minutos) durante quatro dias, ajudando a rápida redução de uma dose mais alta para uma dose menor, sendo o protocolo do Flumazenil 
igual a todos os pacientes, sem diferença entre sexo, idade, índice de massa corporal e ingestão diária de BZDs (Brett \& Murnion, 2015).

\section{Considerações Finais}

Apesar das recomendações do uso de BZDs a curto prazo, o uso prolongados em idosos ainda é prática comum na terapeutica, principalmente os BZDs de longa ação, que não são recomendados para o uso em idosos, devido as alterações fisiológicas decorrentes do envelhecimento e que alteram na farmacocinética do fármaco, essas mudanças resultam em metabolitos ativos que ficam por mais tempo no organismo, acumulando-se nos tecidos adiposos, prolongando a meia vida do fármaco e resultando em efeitos toxicológicos não desejáveis, como a dependência, que pode gerar tolerância aos efeitos do medicamento.

Conclui-se que mesmo os BZDs de curta ação, se utilizados além do recomendado pelas diretrizes de saúde, podem apresentar efeitos toxicológicos, sendo umas delas a dependência química. Para o manejo da dependência os estudos apontam para várias alternativas, porém algumas apresentam efeitos adversos secundarios, superando os efeitos terapeuticos desejados para o tratamento. Muitos estudos apontam como tratamento para a dependencia no uso do Diazepam, que é um BZDs de ação longa, porém sendo a dose diminuída semanalmente, para que assim os efeitos de abstinência não sejam tão sentidos pelos pacientes, o uso da Carbamazepina como adjuvante na retirada dos BZDs é uma proposta viável de acordo com nossa revisão, outro fármaco é o Flumazenil que além de reduzir os sintomas de abstinência, podem controlar a tolerância.

Por tanto, a retirada do uso dos BZDs de pacientes com uso a longo prazo é complicada pelo aparecimento de sintomas em que muitas das vezes podem ser confundidas com o aparecimento das causas inicias para o uso dos BZDs, fazendo com que o paciente retorne ao uso novamente. Por isso é interessante a diminuição gradual utilizando um fármaco de longa ação ou com outro um adjuvante, para que seja controlado os possíveis sintomas de abstinencia. Por fim, sugere-se que sejam feitos estudos de que forma se dá o mecanismo da tolerância dos benzodiazepínicos para que assim possam ser identificadas formas evitar esses efeitos. Espera-se que com a evolução da farmacoterapia e aos avanços da farmacocinética e farmacodimica no futuro possamos melhor entender os mecanismos de tolerância entre fármacos/receptores.

\section{Referências}

Alvarenga, J. M., Giacomin, K. C., Filho, A. I. de L., Uchoa, E. \& Firmo, J. O. A. (2014). Chronic use of benzodiazepines among older adults. Revista de Saúde Pública, 48(6), 866-872.

Baandrup, L., Ebdrup, B. H., Rasmussen, J. O., Lindschou, J., Gluud, C. \& Glenthoj, B. Y (2018). Pharmacological interventions for benzodiazepine discontinuation in chronic benzodiazepine users. Cochrane Database Of Systematic Reviews, 2018(3), 1-159.

Benini, A., Gottardo, R. Chiamulera, C. BertoldI, A., Zamboni, L. \& Lugoboni, F. (2021). Continuous Infusion of Flamazenil in the Management of Benzodiazepines Detoxification. Front Psychiatry, 12, 1-7.

Botelho, L. L. R., Cunha, C. C. de A. \& Macedo, M. (2011). O método da revisão integrativa nos estudos organizacionais. Revista Eletronica Gestão e Sociedade, 5(11), 121-136.

Brett, J. \& Murnion, B. (2015). Management of benzodiazepine misuse and dependence. Australian Prescriber, $38(5), 152-155$.

Calcaterra, N. E. \& Barros, J. C. (2014). Classics in chemical neuroscience: diazepam (valium). ACS Chemical Neuroscience, 5(4), $253-260$.

Canham, S. L., Gallo, J. \& Simoni-Wastila, L. (2014). Perceptions of benzodiazepine dependence among women age 65 and older. Journal Of Gerontologia Social Work, 57(8), 872-888.

Cunha, C. D. dos A., Souza, M. C. C., Cattanio, G. A. A., Iahnn, S. R. \& Lima, R. da C. (2015). Benxodiazepine use and assoociated factors in elderly in the city of Dourados, MS, Brazil. Jornal Brasileiro de Psiquiatria, 64(3), 207-212.

Davies, E. A. \& O’Mahony, M. S. (2015). Adverse drug reactions in special populations - the elderly. British Journal Of Clinical Pharmacology, 80(4), 796807.

Danza, A., Branco, M. R., Pampín, M. L., Agorio, D., Caleri, A., Patiño, I., Casal, M. \& Días, L. (2015). Benzodiazepinas y fractura de cadera: estúdio de casos y controles. Rev. Méd. Urug, Montevidéu, 31(2), 120 - 127. 
Domínguez, V., Collares, M., Ormaechea, G. \& Tamosiunas, G. et al. (2016). Uso racional de benzodiacepinas: hacia uma mejor prescripción. Rev. Urug, Med. Int., Montevidéu, 1(3), 14-24.

Filho, P. C. P. T., Chagas, A. R., Pinheiro, M. L. P., Lima, A. M. de J. \& Durão, A. M. S. (2011). Utilização de benzodiazepínicos por idosos de uma estratégia de saúde da família: implicações para enfermagem. Escola Anna Nery, 15(3), 581-586.

Fluyau, D., Revadigar, N. \& Manobianco, B. E. (2018). Challenges of the pharmacological management of benzodiazepine withdrawal, dependence, and discontinuation. Therapeutic Advances In Psychopharmacology, 8(5), 147-168.

Gage, S. B., Moride, Y., Ducruet, T. Kurth, T., Verdoux, H., Tournier, M. Pariente, A. \& Bégaud, B. (2014). Benzodiazepine use and risk of Alzheimer's disease: case-control study. BMJ, 349(092), g5205.

Gage, S. B., Bégaud, B., Bazin, F., Verdoux, H., Daetigues, J. F., Pérès, K., Kurth, T. \& Pariente, A. (2012). Benzodiazepine use and risk of dementia: prospective population based study. BMJ, 345(274), e6231.

Griffen, C. E., Kaye, M. A., Bueno, F. R. \& Kaye, L. D. (2013). Benzodiazapine pharmacology and central nervos system-mediadted effectes. Ochsner J, 13(2), 214-233.

Hood, S. D., Norman, A., Hince, D. A., Melichar, J. K. \& Hulse, G. K. (2014). Benzodiazepine dependesse and its treatment with low dose flumazenil. British Journal Clinical Pharmacology, 77(2), 285-294.

Jacob, L., Raapp, M. A. \& Kostev, K. (2017). Long-term use of benzodiazepines in older patientis in Germany: a retrospective analysus. Therapeutic Advances In Psychopharmacology, 7(6-7), 191-200.

Janhser, K., Roser, P. \& Hoffmann, K. (2015). The Problems of Long-Term Treatment With Benzodiazepines and Related Substances. Deutsches Aerzteblatt Online, 12(1-2), 1-7.

Jiang, Y., Xia, Q., Wang, J., Zhou, P., Jiang, S., Diwan, V. K. \& Xu, B. (2019). Insomnia, Benzodiazepine Use, andFalls among Residents in Long-term Care Facilities. International Journal Of Environmental Research and Public Health, 16(23), p. 1-11.

Kang, M., Galuska. M. A. \& Ghassemzadh, S. (2021) Benzodiazepine Toxicity. In: StartPearls. Treasure Island (FL); StatPearl Publishing. Pubmed.ncbi.nlm.nih.gov/29489152/

Katzung, B. G., Masters, S. B. \& Trevor, A. J. (2014). Farmacologia básica e clínica. (12a ed.), AMGH.

Lader, M. (2014). Benzodiazepine harm: how can it be reduced?. BR J Clin Pharmacol, 77(2), 295- 301.

Marsden, J., White, M., Annand, F., Burkinshaw, P., Carville, S., Eastwood, B., Kelleher, M., Knight, J., O’Connor, R., Tran, A., Willey, P., Greaves, F. \& Taylor, S. (2019). Medicines associated with dependence or withdrawal: a mixed-methods public health review and national database study in england. The Lancet Psychiatry, 6(11), 935-950.

Neft, M. W., Oerther, S., Halloway, S., Hanneman, S. K. \& Mitchell, A. M. (2019). Benzodiazepine and antipsychotic medication use in older adults. Nurs Open. Nursing Open, 7(1), 4-6.

Olsen, R. W. (2018). GabaA receptor: Positive and negativo allosteric modulators. Neuropharmacology, 136(Pt A),10-22.

Pomara, N., Lee, S. H., Bruno, D., Silber, T., Greenclatt, D. J., Petkova, E. \& Sidtis, J. J. (2015). Adverse performance effects of acute lorazepam administration in elderly long-term users: pharmacokinetic and clinical predictors. Progress In NEuro-Psychopharmacology And Biological Psychiatry, 56, $129-135$.

Schallember, J. B. \& Colet, C. F. (2016). Assessmet of dependence and anxiety among benzodiazepine users in a provincial municipality in Rio Grande do Sul, Brazil. Trends In Psychiatry and Psychotherapy, 38(2), 63-70.

Reeves, R. R. \& Kamal, A. (2019). Complicated Withdrawal Phenomena During Benzodiazepine Cessation in Older Adults. Journal Of Osteopathic Medicine, $119(5), 327-331$.

Rosas-Gutierrez, I., Simon-Arceo, K. \& Mercado, F. (2013). Mecanismo celular y molecular de la adiccion a benzodiacepinas. Salud Ment, 36(4), 325-329.

Tajiri, K. \& Shimizu, Y. (2013). Liver physiology and liver diseases in the elderly. World Journal Of Gastroenterology, $19(46)$, 8459-8467.

Torres-Bondia, F., Batller, J. de, Galván, L., Buti, M., Barbé, F. \& Piñol-Ripoll, G. (2020). Trends in the consumumption rates of benzodiazepines and benzodiazepine-related drugs in the health region of Lleida from 2002 to 2015. BMC public health, 20(1), 1-9.

Tseng, L. Y., Huang, S. T., Peng, L. N., Chen, L. K. \& Hsiao, F. Y. (2019). Benzodiazepines, z-Hypnotics, and Risk of dementia: Special Considerations of Half-Lives and Concomitant Use. Neurotherapeutics: the jornal of the American Society for Experimental Neuro Therapeutics, 17(1), $156-164$.

Vila, J. V., Vila, M. D. M. V., Ibáñez, L. S., Zaragoza, J. A. A. \& Royo, L. M. (2012). Adecuación de la utilización de benzodiazepinas em anciano desde la oficina de farmácia. Um estúdio de colaboración médico-farmacéutico. Atención Primaria, 44(7), 402-410.

Vinckenbosch, F. R. J., Vermeeren, A., Vuurman, E. F. P. M., Sluiszen, N. N. J. J. M. D., Verster, J. C., Loo, A. J. A. E. V., Dijken, J. H. V., Veldstra, J. L., Brookhuis, K. A., Waard, D. \& Ramaekers, J. G. (2021). Na explorative approach to understanding individual diferences in driving performance and neurocognition in long-term benzodiazepine users. Human Psychopharmacology: Clinical and Experimental, 36(4), 1-17.

Vinkers, C. H. \& Olivier, B. (2012). Mechanisms Underlying Tolerance after Long-Term Benzodiazepine Use: A Future for Subtype-Selective GABA (A) Receptor Modulators?. Advances In Pharmacological Sciences, 2012, 1-19. 